\title{
Coprecipitation Behaviors of Zr, Hf, and Th with Sm Hydroxide for Chemical Study of Element 104, Rf
}

\author{
Y. Kasamatsu, ${ }^{*, a}$ K. Toyomura, ${ }^{\text {a }}$ N. Shiohara, ${ }^{\text {a }}$ T. Yokokita, ${ }^{\text {a }}$ Y. Komori, ${ }^{a}$ A. Kino, ${ }^{\text {a }}$ T. Yoshimura, ${ }^{\text {b }}$ \\ N. Takahashi, ${ }^{a}$ H. Haba, ${ }^{\mathrm{c}}{ }^{\text {Y. Kudou},}{ }^{\mathrm{c}}$ H. Kikunaga, ${ }^{\mathrm{d}}$ T. Mitsugashira, ${ }^{\mathrm{e}}$ T. Ohtsuki, ${ }^{\mathrm{f}}$ K. Takamiya, ${ }^{\mathrm{f}}$ \\ and A. Shinohara ${ }^{\mathrm{a}}$ \\ ${ }^{\mathrm{a}}$ Graduate School of Science, Osaka University, 1-1 Machikaneyama, Toyonaka, Osaka 560-0043, Japan \\ ${ }^{\mathrm{b}}$ Radioisotope Research Center, Osaka University, 2-4 Yamadaoka, Suita, Osaka 565-0871, Japan \\ ${ }^{\mathrm{c} N i s h i n a}$ Center for Accelerator-Based Science, RIKEN, Wako, Saitama 351-0198, Japan \\ ${ }^{\mathrm{d}}$ Research Center for Electron Photon Science, Tohoku University, 1-2-1 Mikamine, Taihaku-ku, Sendai, Miyagi \\ 982-0826, Japan \\ 'Institute for Material Research, Tohoku University, Oarai, Ibaraki 311-1313, Japan \\ ${ }_{\mathrm{f}}^{\mathrm{f}}$ Research Reactor Institute, Kyoto University, Kumatori-cho, Sennan-gun, Osaka 590-0494, Japan
}

Received: February 25, 2014; In Final Form: July 7, 2014

\begin{abstract}
In superheavy element chemistry, rapid single-atom chemistry combined with rapid identification via alpha-particle measurement is required to be performed. Herein, we propose a new protocol for rapid preparation of coprecipitates of superheavy elements with Sm hydroxide. The coprecipitation behaviors of Zr, Hf, and Th (homologues of element 104, Rf) with Sm hydroxide in aqueous ammonia $\left(\mathrm{NH}_{3}\right)$ and sodium hydroxide $(\mathrm{NaOH})$ solutions were investigated by the conventional suction filtration method using ${ }^{88} \mathrm{Zr},{ }^{175} \mathrm{Hf}$, and ${ }^{228} \mathrm{Th}$ radiotracers. The precipitation yields obtained by employing an aging time of only $10 \mathrm{~s}$ were consistent with those obtained after aging for 10 min, suggesting the rapid chemical reactions in the present coprecipitation. Approximately 100\% coprecipitation yields of $\mathrm{Zr}$ and $\mathrm{Hf}$ were achieved in the solutions with dilute hydroxide concentrations, and the yields decreased with higher hydroxide concentrations. In comparison, the Th yield remained constant at almost $100 \%$ under all of the conditions. In addition, a semiautomatic suction filtration apparatus for rapid preparation of precipitated samples was developed and evaluated using the short-lived isotopes ${ }^{89 \mathrm{~m}, \mathrm{~g}} \mathrm{Zr}$ and ${ }^{173} \mathrm{Hf}$, which were transported online from the nuclear reaction chamber by a $\mathrm{He} / \mathrm{KCl}$ gas-jet system. Using this apparatus, sample preparation was completed within $80-120 \mathrm{~s}$, and precipitation yields consistent with those determined on the basis of the manual method were obtained, suggesting the applicability of the present method to Rf experiment.
\end{abstract}

\section{Introduction}

The influence of relativistic effects on electron orbital shells becomes significant for heavy elements because of the higher positive charges on their nuclei. Therefore, the chemical properties of the transactinide elements with atomic numbers $Z \geq$ 104 , i.e., superheavy elements, are expected to deviate from the characteristic periodicity of the lighter homologues in the periodic table, and their chemical studies have attracted much attention. ${ }^{1,2}$ However, experimental evaluation of these species is fraught with difficulties. Because of the extremely low production rates and short half-lives (within a few min) of these heavy nuclides, chemical evaluations of the superheavy elements must be conducted on a one-atom-at-a-time basis, ${ }^{3}$ and must be rapidly completed. Thus, simple chemistry employing partition methods such as solvent extraction and ion-exchange chromatography have been utilized and the chemical separations were repeated hundreds or thousands of times under identical conditions. This approach requires two types of apparatuses: an online system having the capability for rapid transport of nuclear reaction products from the reaction chamber to the chemistry laboratory, and a rapid chemistry apparatus equipped with a radiation detection system. ${ }^{1,2}$ Additionally, for unambiguous identification of superheavy elements on a one-atom-at-a-time basis, it is necessary to measure the energies and lifetimes of $\alpha$ or sometimes spontaneous fission decays. For these measurements using a Si detector, the sample

*Corresponding author. E-mail: kasa@chem.sci.osaka-u.ac.jp TEL: +81-6-6850-5418; FAX: +81-6-6850-6999 must be dried and flat without salt contaminants. Liquid scintillation counting is an alternative analytical approach, but the energy resolution of this technique is very poor compared with that using the Si detector. Moreover, $\alpha$-particle measurement using liquid scintillation is easily disturbed by $\beta$ - and $\gamma$-rays. Above-mentioned experimental difficulties limit the variety of chemical investigation of superheavy elements.

Thus far, primarily on the basis of gas-phase and aqueousphase chromatographic experiments, transactinide elements with $Z$ up to 108 have been assigned as $d$-block metal elements belonging to the 7 th period of the periodic table. ${ }^{1,2}$ Experiments employing solution chemistry have focused mainly on the lightest transactinide element, rutherfordium (Rf, $Z=104) .{ }^{4-10}$ On the basis of the similarity of the behavior of Rf to the homologues $\mathrm{Zr}$ and $\mathrm{Hf}$, these experiments revealed that $\mathrm{Rf}$ is a group 4 element. More recently, detailed ion-exchange experiments of Rf have been succeeded, yielding interesting results; the anion- and cation-exchange behaviors of Rf in HF and HF/ $\mathrm{HNO}_{3}$ solutions are clearly different from those of the homologues $\mathrm{Zr}$ and Hf. ${ }^{11-13}$ In particular, the distribution coefficients of $\mathrm{Rf}$ in the cation exchange lay between those of the homologues and the pseudo-homologue Th. ${ }^{13}$ Detailed studies on Rf in various chemical systems are still required to clarify the chemical properties of Rf, which leads to more in-depth understanding of the characteristic chemical properties of superheavy elements.

The purpose of the present study is to establish a new experimental methodology for investigating the chemical properties of superheavy elements, specifically, the coprecipitation properties of ${ }^{261} \operatorname{Rf}\left(T_{1 / 2}=68 \mathrm{~s}\right){ }^{14}$ by the formation of hydroxide and 
ammine complexes. Coprecipitation technique has been widely used for chemical separation and so on in radiochemical researches, and the coprecipitation is one of the important chemical properties. ${ }^{15,16}$ In general, however, the energy resolution of a precipitate sample in the $\alpha$ spectrum is poor, and indeed, the precipitation method has not yet been applied to superheavy element chemistry. For this problem, we successfully established a simple coprecipitation method with Sm hydroxide, which facilitates $\alpha$ spectrometry with high energy resolution. ${ }^{17}$ Herein, this method was applied to the investigation of the coprecipitation behaviors of $\mathrm{Zr}$, Hf, and Th with Sm hydroxide. In addition, a semiautomatic apparatus for repetitive preparation of precipitate samples was developed. Experiments utilizing the apparatus connected to the gas-jet transport system from the nuclear reaction chamber were performed on ${ }^{89 \mathrm{~m}, \mathrm{~g}} \mathrm{Zr}$ and ${ }^{173} \mathrm{Hf}$ samples produced by the accelerator as a model experiment for ${ }^{261} \mathrm{Rf}$.

\section{Experimental}

2.1. Coprecipitation experiment for $\mathrm{Zr}$, $\mathrm{Hf}$, and $\mathrm{Th}$ with Sm hydroxide. Carrier-free radiotracers of ${ }^{88} \mathrm{Zr}\left(T_{1 / 2}=83.4 \mathrm{~d}\right)$ and ${ }^{175} \mathrm{Hf}(70.0 \mathrm{~d})$ were produced in the nuclear reactions of ${ }^{89} \mathrm{Y}(p, 2 n){ }^{88} \mathrm{Zr}$ and ${ }^{175} \mathrm{Lu}(p, n){ }^{175} \mathrm{Hf}$, respectively, using the AVF cyclotron at the Research Center of Nuclear Physics (RCNP), Osaka University. The proton beam energy was $22 \mathrm{MeV}$ and the irradiation time was ca. $1 \mathrm{~h}$ for the production of ${ }^{88} \mathrm{Zr}$. For ${ }^{175} \mathrm{Hf}$, the corresponding parameters were $14 \mathrm{MeV}$ and $7 \mathrm{~h}$. The beam current was $\sim 1 \mu \mathrm{A}$ for both irradiations. The nuclides were then purified by chemical separation protocols similar to those in Reference 18. The radiotracer ${ }^{228} \mathrm{Th}(1.9 \mathrm{y})$ was chemically separated from its grandmother nuclide ${ }^{228} \mathrm{Ra}(5.75 \mathrm{y})$ as follows. First, $8 \mathrm{M}$ nitric acid $\left(\mathrm{HNO}_{3}\right)$ containing ${ }^{228} \mathrm{Th},{ }^{228} \mathrm{Ra}$, and other daughter nuclides was fed onto the strongly basic anion-exchange column (Muromac $1 \times 8,200-400$ mesh, 5$\mathrm{mm}$ i.d. $\times 15-\mathrm{mm}$ height, $\mathrm{NO}_{3}{ }^{-}$form). ${ }^{228} \mathrm{Ra}$ and other nuclides, except for ${ }^{228} \mathrm{Th}$ and some relatively short-lived daughter nuclides, were then eluted with $8 \mathrm{M} \mathrm{HNO}_{3}$. After decay of those short-lived nuclides, the column was washed with $8 \mathrm{M}$ $\mathrm{HNO}_{3}$, and ${ }^{228} \mathrm{Th}$ was subsequently eluted with $2 \mathrm{M}$ hydrochloric acid $(\mathrm{HCl})$. This separation was repeated to purify ${ }^{228} \mathrm{Th}$. After completion of the chemical purification, the ${ }^{88} \mathrm{Zr},{ }^{175} \mathrm{Hf}$, and ${ }^{228} \mathrm{Th}$ radiotracers were mixed together in $0.01 \mathrm{M} \mathrm{HCl}$ and used in the following coprecipitation experiment.

Coprecipitated samples with Sm hydroxide were prepared using various basic solutions such as dilute and concentrated aqueous $\mathrm{NH}_{3}$ and $0.1,1.0,6.0$, and $12.0 \mathrm{M} \mathrm{NaOH}$ solutions. A $20 \mu \mathrm{L}$ aliquot of the Sm standard solution (Sm $1000 \mathrm{ppm}, 1 \mathrm{M}$ $\mathrm{HNO}_{3}$ ) was added into $220 \mu \mathrm{L}$ of an aqueous solution $(\sim 0.4 \mathrm{M}$ $\mathrm{HCl})$ containing the radiotracers in a polypropylene (PP) beaker. The solution was stirred and $2 \mathrm{~mL}$ of the basic solution was then added to produce the precipitate. When the dilute aqueous $\mathrm{NH}_{3}$ was used, we checked the basicity of the solution using a $\mathrm{pH}$-test paper. After aging the precipitate for $10 \mathrm{~min}$ at $25^{\circ} \mathrm{C}$, the precipitate sample was subjected to suction filtration with a PP membrane filter (eichrom, Resolve ${ }^{\circledR}$ Filters $0.1 \mu \mathrm{m}$ ). The obtained sample was dried on a heater at $100{ }^{\circ} \mathrm{C}$, and was subjected to $\gamma$-ray and $\alpha$-particle measurements. The experiments with the aging time of $10 \mathrm{~s}$ were also performed at $25^{\circ} \mathrm{C}$ to determine suitable experimental conditions for Rf. The radioactivities of the filtrate and $\mathrm{PP}$ beakers used were also measured. The standard radioactivities of ${ }^{88} \mathrm{Zr},{ }^{175} \mathrm{Hf}$, and ${ }^{228} \mathrm{Th}$ were determined at the same detection efficiency by preparing standard samples with the same diameter as the precipitate sample. In the present study, we define a precipitation yield, $Y$, and the ratio of adsorption on the PP beaker, $R_{\mathrm{ad}}$, according to eqs 1 and 2, respectively.

$$
\begin{aligned}
& Y=\frac{A_{\mathrm{ppt}}}{A_{\text {std }}} \times 100 \\
& R_{\text {ad }}=\frac{A_{\text {cup }}}{A_{\text {ppt }}+A_{\text {fil }}+A_{\text {cup }}} \times 100
\end{aligned}
$$

In the above equations, $A_{\mathrm{ppt}}, A_{\text {fil }}$, and $A_{\text {cup }}$ represent the radioactivities of the precipitate, filtrate, and PP beaker used, respectively, and $A_{\text {std }}$ represents the standard radioactivity. The errors in the yields were determined by taking into account the standard deviation in each $\gamma$ or $\alpha$ count $(0.4-6.3 \%)$ and in the variation of the precipitation yield (5\%). It is difficult in superheavy element chemistry to determine the $A_{\text {fil }}$ value by rapidly measuring $\alpha$-activity of the filtrate containing salt. Thus, we decided to use the $Y$ value which is a relative value to the standard radioactivity, whereas the $A_{\text {std }}$ value is required to be separately determined. The $Y$ value can be determined even for short-lived nuclides. However, the $Y$ value would show a lower value if physical adsorption of the complex is strong. The $R_{\mathrm{ad}}$ value was, therefore, determined to evaluate the effect of the physical adsorption of the $\mathrm{Zr}$, Hf, and Th complexes on the PP beaker on the analytical result. For Th, $R_{\text {ad }}$ was determined by evaluating the ratio of $A_{\text {ppt }}$ to $A_{\text {std }}$ because $A_{\text {fil }}$ of Th could not be determined by $\alpha$-particle measurement because of interference from $\mathrm{NaCl}$ and/or $\mathrm{NH}_{4} \mathrm{Cl}$ salts in the $\alpha$ spectrometry.

The energy resolution of the $5423-\mathrm{keV} \alpha$ peak of ${ }^{228} \mathrm{Th}$ was measured from the full width at half maximum (FWHM) for each precipitate sample. The distance between the Si detector and the sample was $\sim 20 \mathrm{~mm}$.

2.2. Online precipitation experiment using the apparatus. A computer-controlled suction filtration apparatus for the preparation of precipitated samples of heavy elements (termed CHIN based on the Japanese word for precipitate, "CHINDEN") was developed using cylinders operated by compressed air, valves operated by on/off operation of $24 \mathrm{~V} \mathrm{DC}$ power and so on. A compact data acquisition system (CompactDAQ) and LabVIEW software from National Instruments Corporation were used for the operation of the apparatus. The suction filtration part of the apparatus and simple movement are schematically illustrated in Figure 1. This apparatus performs rapid and repetitive filtration. Filter sticks were fabricated using polytetrafluoroethylene (PTFE) to prevent the adsorption of the radiotracers on the wall of the stick, and the stick was washed after each usage.

The online experiment using CHIN was performed with relatively short-lived nuclides ${ }^{89 \mathrm{~m}, \mathrm{~g}} \mathrm{Zr}(4.2 \mathrm{~min}$ and $78.4 \mathrm{~h})$ and ${ }^{173} \mathrm{Hf}(23.6 \mathrm{~h})$ produced using the AVF cyclotron at RCNP. These nuclides produced in the reactions of ${ }^{\text {nat }} \operatorname{Sr}(\alpha, x n)^{89 \mathrm{~m}, \mathrm{~g}} \mathrm{Zr}$ and ${ }^{\text {nat }} \mathrm{Yb}(\alpha, x n){ }^{173} \mathrm{Hf}$ were rapidly and continuously transported from the nuclear reaction chamber to the chemistry laboratory using the $\mathrm{He} / \mathrm{KCl}$ gas-jet system in the same manner employed in superheavy element chemistry. ${ }^{1,2}$ The products were collected on the collection site of the dissolution apparatus. After dissolution by $200 \mu \mathrm{L}$ of dilute $\mathrm{HCl}$ for $12 \mathrm{~s}$, the precipitate samples were prepared at room temperature in a manner similar to that used in the tracer experiment described above, with stirring for $10 \mathrm{~s}$. The samples were filtered with the filtration apparatus, and were subsequently dried on a heater under hot air. The coprecipitation yields were determined from the $\gamma$-ray radioactivity of the precipitated sample relative to the total radioactivity of the precipitated and filtrate samples.

\section{Results and discussion}

3.1. Coprecipitation experiment for $\mathrm{Zr}$, $\mathrm{Hf}$, and $\mathrm{Th}$ with Sm hydroxide. The hydroxide ion concentrations of six solutions prepared by the addition of dilute and concentrated aqueous $\mathrm{NH}_{3}$ and $0.1,1.0,6.0$, and $12.0 \mathrm{M} \mathrm{NaOH}$ solution were 


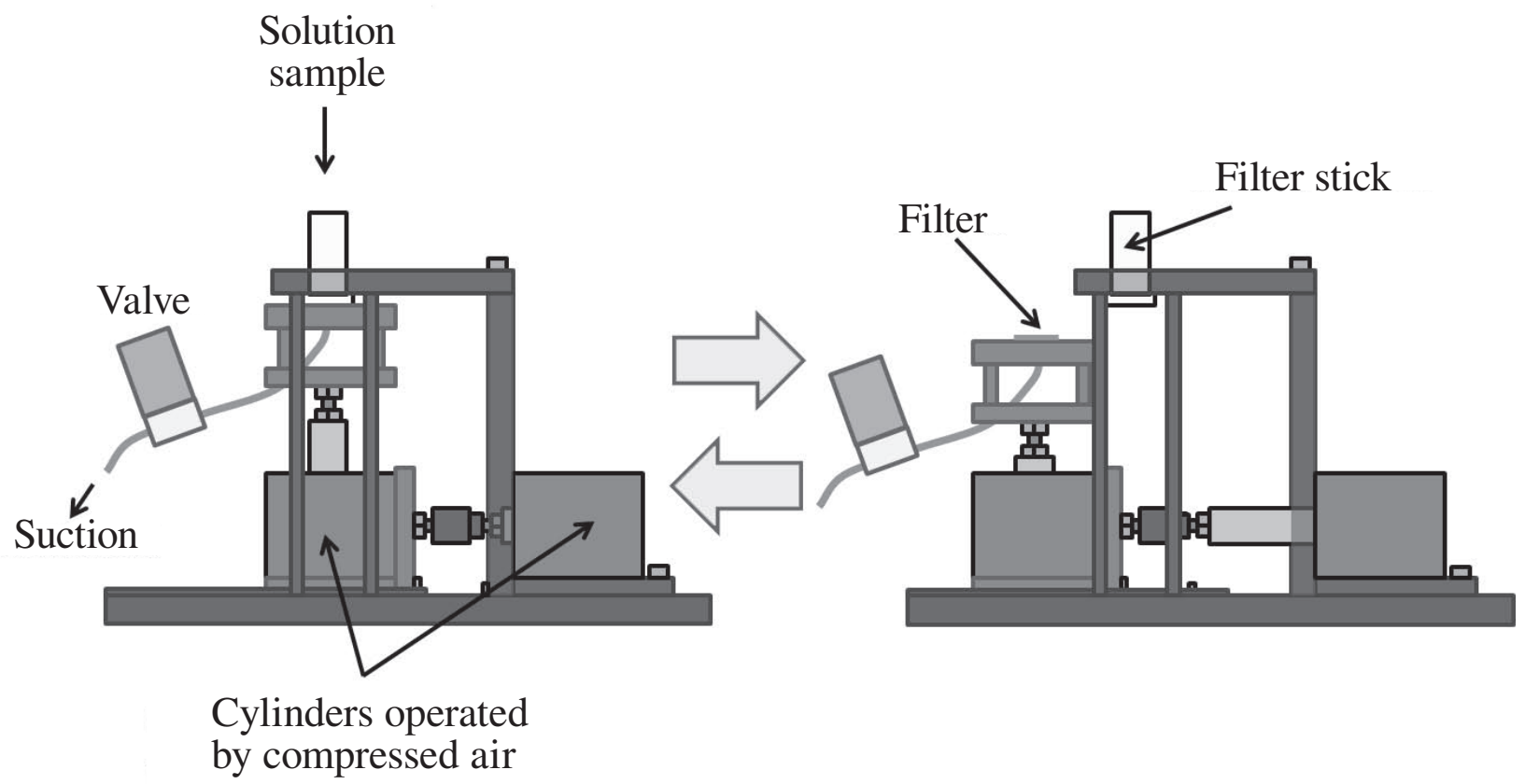

Figure 1. Schematic illustration of the filtration section of rapid suction filtration apparatus.

calculated as $2.3 \times 10^{-3}, 0.016,0.079,0.84,5.3$, and $11 \mathrm{M}$, respectively, using the dissociation constant of aqueous $\mathrm{NH}_{3} .{ }^{19}$ The concentration of $\mathrm{NH}_{3}$ in the samples prepared by adding dilute and concentrated aqueous $\mathrm{NH}_{3}$ were 0.28 and $13 \mathrm{M}$, respectively. The $R_{\text {ad }}$ values are shown in Table 1 . The ratio of physical adsorption of $\mathrm{Zr}$, Hf, and Th on the surface of the PP beaker for samples aged for $10 \mathrm{~s}$ was generally less than $3 \%$, and was slightly lower than those of samples aged for $10 \mathrm{~min}$. These low adsorption ratios especially for the samples aged for $10 \mathrm{~s}$ suggests that physical adsorption of these group 4 elements during coprecipitation with Sm hydroxide was small enough to be negligible in the determination of the coprecipitation yields. The coprecipitation yields of $\mathrm{Zr}, \mathrm{Hf}$, and $\mathrm{Th}$ when 10-min aging was employed are shown in Figures 2(a), and those obtained with 10-s aging are displayed in Figures 2(b). Figures 2(a) and (b) demonstrate that the yields obtained with 10 -min and 10-s aging are in good agreement. This suggests that the chemical reactions during coprecipitation of $\mathrm{Zr}$ and $\mathrm{Hf}$ are fast, and the present conditions are suitable for evaluating the coprecipitation behavior of ${ }^{261} \mathrm{Rf}$ with $T_{1 / 2}=68 \mathrm{~s}$. Based on this result and the small adsorption especially for 10-s aging, it was found that accurate precipitation yields of the group 4 elements could be obtained under the present conditions by the rapid method.

Almost complete coprecipitation of $\mathrm{Zr}$ and $\mathrm{Hf}$ with $\mathrm{Sm}$ hydroxide was achieved when aqueous $\mathrm{NH}_{3}$ solutions and 0.1
$\mathrm{M} \mathrm{NaOH}$ were used. These results are consistent with the wellknown properties of these elements, i.e., hydroxide precipitation in basic solutions. ${ }^{20,21}$ With the use of more highly concentrated $\mathrm{NaOH}$ solutions, the yields of $\mathrm{Zr}$ and $\mathrm{Hf}$ decreased as the $\mathrm{OH}^{-}$concentration increased, whereas the Th yields were constant at ca. $100 \%$. This is attributed to the fact that $\mathrm{Zr}$ and $\mathrm{Hf}$ form hydroxide complex ions such as $\left[\mathrm{M}(\mathrm{OH})_{5}\right]^{-}\left(\mathrm{M}^{4+}=\right.$ $\mathrm{Zr}^{4+}$ and $\left.\mathrm{Hf}^{4+}\right)^{22,23}$ in solutions with high hydroxide concentration. Indeed, we also observed the decrease of the precipitate of $\mathrm{Zr}$ and $\mathrm{Hf}$ on the macro scale in concentrated hydroxide solutions. The yields of Hf were consistently lower than those of $\mathrm{Zr}$ in the same solutions. This is qualitatively consistent with the larger solubility product of $\left[\mathrm{Hf}(\mathrm{OH})_{5}\right]^{-}\left(\log K_{\mathrm{s} 5}=-3.2\right)$ than that of $\left[\mathrm{Zr}(\mathrm{OH})_{5}\right]^{-}\left(\log K_{\mathrm{s} 5}=-3.6\right){ }^{22}$ whereas that of $\mathrm{Th}$ is quite small $\left(\log K_{\mathrm{s} 5}=-5.8\right){ }^{22}$ The $\sim 100 \%$ coprecipitation yields of $\mathrm{Zr}$, Hf, and $\mathrm{Th}$ with the use of aqueous $\mathrm{NH}_{3}$ indicate that the ammine-coordinated complex ions, which dissolve in aqueous solution (e.g., $\mathrm{Zn}, \mathrm{Cu}$, and $\mathrm{Ag}$ hydroxide dissolve in concentrated $\mathrm{NH}_{3}$ solutions), are not dominantly formed even in concentrated $\mathrm{NH}_{3}$ solution. On the basis of coprecipitation experiments with Sm hydroxide using various radioactive lanthanide nuclides, it was confirmed beforehand that the yields of Sm are almost constant around $100 \%$ under all of the conditions studied. The obtained results indicate that the hydroxide and ammine complexation properties of group 4 elements can be qualitatively investigated through their coprecipitation

TABLE 1: Adsorption ratios of $\mathrm{Zr}$, Hf, and Th on polypropylene beakers

\begin{tabular}{|c|c|c|c|c|c|c|}
\hline \multirow{3}{*}{$\begin{array}{l}\text { Added basic } \\
\text { solution }\end{array}$} & \multicolumn{6}{|c|}{ Adsorption ratio, $R_{\mathrm{ad}} / \%$} \\
\hline & \multicolumn{3}{|c|}{ 10-min aging } & \multicolumn{3}{|c|}{ 10-s aging } \\
\hline & $\mathrm{Zr}$ & Hf & Th & $\mathrm{Zr}$ & Hf & Th \\
\hline dilute $\mathrm{NH}_{3}$ & $6.4 \pm 1.7$ & $1.6 \pm 1.1$ & $4.1 \pm 0.3$ & $4.3 \pm 1.1$ & $2.6 \pm 1.3$ & $2.7 \pm 0.3$ \\
\hline conc. $\mathrm{NH}_{3}$ & $4.6 \pm 1.3$ & $1.4 \pm 1.0$ & $2.6 \pm 0.3$ & $1.3 \pm 0.6$ & $1.4 \pm 0.9$ & $2.3 \pm 0.3$ \\
\hline $0.1 \mathrm{M} \mathrm{NaOH}$ & $5.0 \pm 1.4$ & $2.2 \pm 1.4$ & $1.7 \pm 0.2$ & $2.1 \pm 1.0$ & $1.4 \pm 1.1$ & $1.2 \pm 0.2$ \\
\hline $1 \mathrm{M} \mathrm{NaOH}$ & $3.5 \pm 0.6$ & $0.4 \pm 1.7$ & $1.8 \pm 0.3$ & $2.5 \pm 0.4$ & $0.5 \pm 1.5$ & $0.7 \pm 0.2$ \\
\hline $6 \mathrm{M} \mathrm{NaOH}$ & $3.8 \pm 0.4$ & $0.5 \pm 1.6$ & $0.7 \pm 0.3$ & $0.5 \pm 0.1$ & $0.3 \pm 1.1$ & N. D. \\
\hline $12 \mathrm{M} \mathrm{NaOH}$ & $4.5 \pm 1.0$ & $0.2 \pm 2.2$ & $0.2 \pm 0.1$ & $1.5 \pm 0.3$ & $0.3 \pm 1.6$ & $0.1 \pm 0.1$ \\
\hline
\end{tabular}


behaviors with $\mathrm{Sm}$ hydroxide under the present experimental conditions.

The FWHM value of the $5423-\mathrm{keV} \alpha$ peak of ${ }^{228} \mathrm{Th}$ for each sample is shown in Table 2 . The energy resolution was adequate for all samples, because the resolution with the present $\mathrm{Si}$ detector is at least $18 \mathrm{keV}$ and our upper acceptable limit is 80 $\mathrm{keV}$ for the identification of ${ }^{261} \mathrm{Rf}$. The resolution for the 10 min aged samples was relatively poor compared to that for the 10 -s aged ones because of the growth of the precipitates during aging.

Overall, it was found that without compromising the yield, well-resolved alpha spectra can be obtained for coprecipitated samples by aging the hydroxide precipitate for only $10 \mathrm{~s}$ at $25^{\circ} \mathrm{C}$. These conditions are all suitable for Rf experiment.

3.2. Online precipitation experiment using the apparatus. The coprecipitation yields obtained in the online experiments are shown in Figure 3. The yields in Figure 3 and their dependence on the composition of the added basic solution are approximately consistent with those in Figure 2(b). This suggests that the present experimental method using CHIN under the present conditions should be applicable to the coprecipitation experiment of Rf. Because the dissolution yield of a product transported by the gas-jet system has some variation, repetitive determination of the coprecipitation yields and averaging are required to obtain more accurate values in the online experiment. In the present online experiment, the time required for the sample preparation from the start of the dissolution was 80-120 s, except in the case employing $12 \mathrm{M} \mathrm{NaOH}$. With the use of $12 \mathrm{M} \mathrm{NaOH}$, more than 4 min was required for the suction filtration because of the high viscosity of the solution; consequently, the use of $12 \mathrm{M} \mathrm{NaOH}$ is unsuitable for $\mathrm{Rf}$ experiment. Based on the present experiment, we were able to determine the conditions for suction filtration, sample drying, etc., for rapid preparation of the precipitate samples. Considering the half-life of ${ }^{261} \mathrm{Rf}$ (68 s), more rapid preparation of the coprecipitate is desirable. Shortening the preparation time by methods such as automating the pump and hot gas operations is now in progress.

\section{Conclusion}

In the present study, coprecipitation with Sm hydroxide was established as a new experimental methodology for superheavy element chemistry. The conditions for the evaluation of the hydroxide coprecipitation properties of element 104, Rf, were determined. The coprecipitation behaviors of the group 4 elements $\mathrm{Zr}$ and $\mathrm{Hf}$ and the pseudo-homologue Th with Sm hydroxide were investigated using radiotracers of ${ }^{88} \mathrm{Zr},{ }^{175} \mathrm{Hf}$, and ${ }^{228} \mathrm{Th}$. It was found that 10 -s aging is sufficient to obtain the equilibrium precipitation yields for these elements. Approximately $100 \%$ yields were achieved for $\mathrm{Zr}$, Hf, and $\mathrm{Th}$ in dilute $\mathrm{NH}_{3}, \mathrm{NaOH}$, and concentrated $\mathrm{NH}_{3}$ solutions, and the yields of $\mathrm{Zr}$ and $\mathrm{Hf}$ decreased at higher $\mathrm{OH}^{-}$concentrations, whereas that of Th was constant at $100 \%$. From these results, the following conclusions are drawn: 1) The $\mathrm{Zr}$ and Hf ions form neutral hydroxide complexes in the basic solutions and form anionic hydroxide complexes in concentrated $\mathrm{NaOH}$ solutions. 2) The pseudo-homologue of Rf, Th shows clearly different behavior from those of $\mathrm{Zr}$ and $\mathrm{Hf}$; almost complete coprecipitation with $\mathrm{Sm}$ hydroxide was achieved under all of the conditions.

In the online model experiment of Rf employing the nuclear reaction products $\mathrm{Zr}$ and $\mathrm{Hf}$, which were transported using the gas-jet system, and semiautomatic suction filtration apparatus CHIN, coprecipitation yields consistent with those obtained in the offline experiments were obtained. It was found that the present conditions, except for the case using $12 \mathrm{M} \mathrm{NaOH}$, are suitable for the $\mathrm{Rf}$ experiment. We believe that the present method can be applied to the evaluation of the formation of

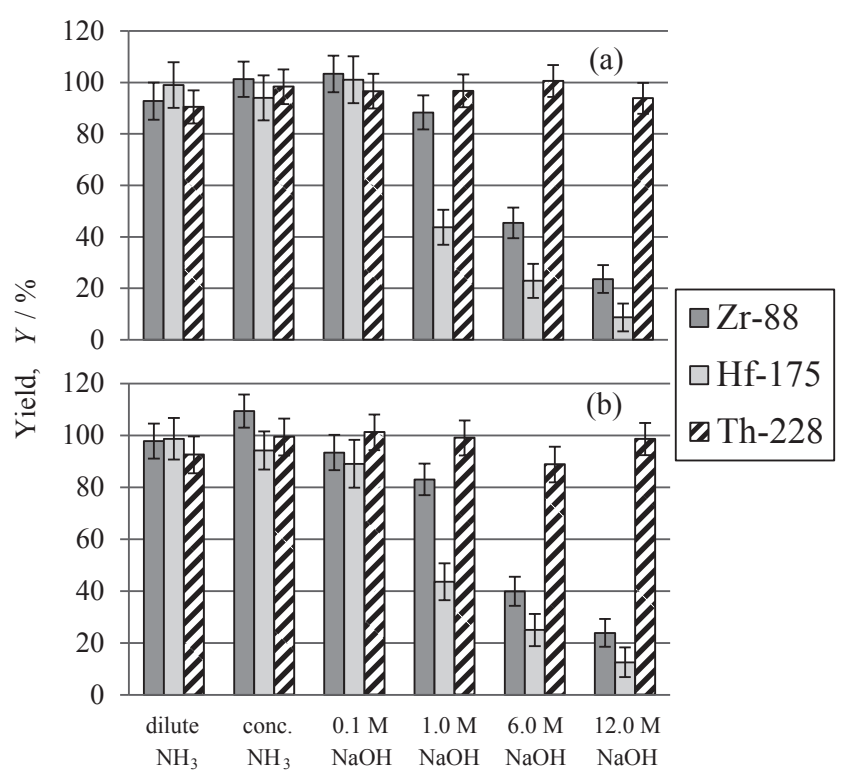

Figure 2. Coprecipitation yields of $\mathrm{Zr}$, Hf, and Th based on the addition of various basic solutions. Yields obtained with 10-min aging are shown in (a) and with 10-s aging in (b).

TABLE 2: Energy resolution of alpha peak for ${ }^{228} \mathrm{Th}$ precipitate samples

\begin{tabular}{ccc}
\hline \multirow{2}{*}{$\begin{array}{c}\text { Added basic } \\
\text { solution }\end{array}$} & \multicolumn{2}{c}{$\begin{array}{c}\text { Energy resolution / keV } \\
\text { at 5423-keV } \alpha \text { peak }\end{array}$} \\
\cline { 2 - 3 } & 10-min aging & 10-s aging \\
\hline dilute $\mathrm{NH}_{3}$ & 29 & 21 \\
conc. $\mathrm{NH}_{3}$ & 46 & 17 \\
$0.1 \mathrm{M} \mathrm{NaOH}$ & 28 & 19 \\
$1 \mathrm{M} \mathrm{NaOH}$ & 32 & 19 \\
$6 \mathrm{M} \mathrm{NaOH}$ & 30 & 18 \\
$12 \mathrm{M} \mathrm{NaOH}$ & 30 & 25 \\
\hline
\end{tabular}

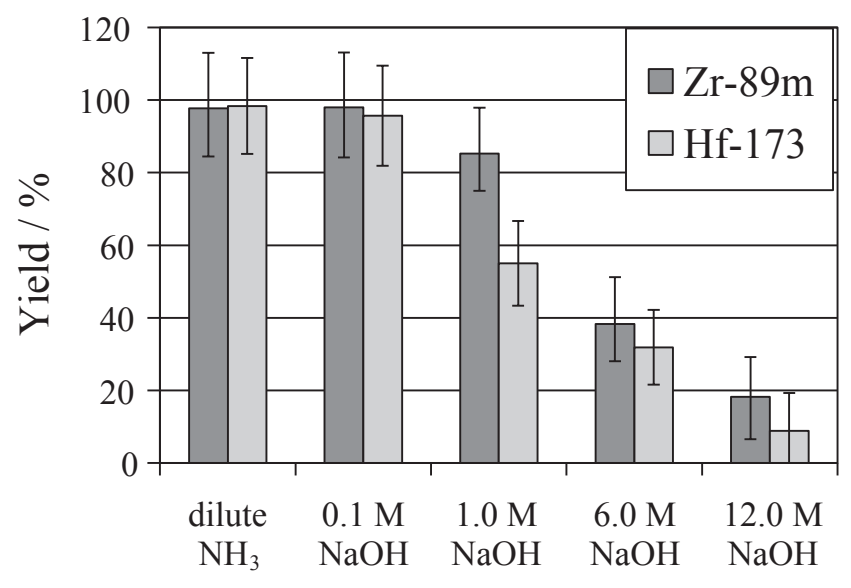

Figure 3. Coprecipitation yields obtained in the online experiment.

ammine and hydroxide complexes of Rf.

\section{Acknowledgement}

We express our gratitude to the staffs of the Accelerator Facility in RCNP for the productions of ${ }^{88,89 \mathrm{~m}, \mathrm{~g}} \mathrm{Zr}$ and ${ }^{173,175} \mathrm{Hf}$. This work was supported by JSPS KAKENHI Grant Number 24655050 . 


\section{References}

(1) The Chemistry of Superheavy Elements, edited by M. Schädel (Kluwer Academic Publishers, Dordrecht, 2003).

(2) M. Schädel, Angew. Chem. Int. Ed. 45, 368 (2006).

(3) R. Guillamont, J. P. Adloff, A. Peneloux, and P. Delamoye, Radiochim. Acta 54, 1 (1991).

(4) H. Haba, K. Tsukada, M. Asai, S. Goto, A. Toyoshima, I. Nishinaka, K. Akiyama, M. Hirata, S. Ichikawa, Y. Nagame, Y. Shoji, M. Shigekawa, T. Koike, M. Iwasaki, A. Shinohara, T. Kaneko, T. Maruyama, S. Ono, H. Kudo, Y. Oura, K. Sueki, H. Nakahara, M. Sakama, A. Yokoyama, J. V. Kratz, M. Schädel, and W. Brüchle, J. Nucl. Radiochem. Sci. 3, 143 (2002)

(5) K. R. Czerwinski, K. E. Gregorich, N. J. Hannink, C. D. Kacher, B. A. Kadkhodayan, S. A. Kreek, D. M. Lee, M. J. Nurmia, A. Türler, G. T. Seaborg, and D. C. Hoffman, Radiochim. Acta 64, 23 (1994).

(6) K. R. Czerwinski, C. D. Kacher, K. E. Gregorich, T. M. Hamilton, N. J. Hannink, B. A. Kadkhodayan, S. A. Kreek, D. M. Lee, M. J. Nurmia, A. Türler, G. T. Seaborg, and D. C. Hoffman, Radiochim. Acta 64, 29 (1994).

(7) C. D. Kacher, K. E. Gregorich, D. M. Lee, Y. Watanabe, B. Kadkhodayan, B. Wierczinski, M. R. Lane, E. R. Sylwester, D. A. Keeney, M. Hendricks, N. J. Stoyer, J. Yang, M. Hsu, D. C. Hoffman, and A. Bilewicz, Radiochim. Acta 75, 127 (1996).

(8) R. Günther, W. Paulus, J. V. Kratz, A. Seibert, P. Thörle, S. Zauner, W. Brüchle, E. Jäger, V. Pershina, M. Schädel, B. Schausten, D. Schumann, B. Eichler, H. W. Gäggeler, D. T. Jost, and A. Türler, Radiochim. Acta 80, 121 (1998).

(9) H. Haba, K. Tsukada, M. Asai, A. Toyoshima, Y. Ishii, H. Toume, T. Sato, I. Nishinaka, T. Ichikawa, S. Ichikawa, Y. Nagame, W. Sato, K. Matsuo, Y. Kitamoto, Y. Tashiro, A. Shinohara, J. Saito, M. Ito, T. Ikezawa, M. Sakamaki, S. Goto, H. Kudo, H. Kikunaga, M. Arai, S. Kamataki, A. Yokoyama, K. Akiyama, K. Sueki, Y. Oura, M. Schädel, W. Brüchle, and J. V. Kratz, Radiochim. Acta 95, 1 (2007).

(10) A. Toyoshima, Y. Kasamatsu, K. Tsukada, M. Asai, Y. Ishii, H. Toume, I. Nishinaka, T. K. Sato, Y. Nagame, M. Schädel, H. Haba, S. Goto, H. Kudo, K. Akiyama, Y. Oura, K. Ooe, A. Shinohara, K. Sueki, and J. V. Kratz, J. Nucl. Radiochem. Sci. 11, 7 (2010).

(11) H. Haba, K. Tsukada, M. Asai, A. Toyoshima, K. Akiyama, I. Nishinaka, M. Hirata, T. Yaita, S. Ichikawa, Y. Nagame, K. Yasuda, Y. Miyamoto, T. Kaneko, S. Goto, S. Ono, T. Hirai, H. Kudo, M. Shigekawa, A. Shinohara, Y. Oura, H.
Nakahara, K. Sueki, H. Kikunaga, N. Kinoshita, N. Tsuruga, A. Yokoyama, M. Sakama, S. Enomoto, M. Schädel, W. Brüchle, and J. V. Kratz, J. Am. Chem. Soc. 126, 5219 (2004).

(12) A. Toyoshima, H. Haba, K. Tsukada, M. Asai, K. Akiyama, S. Goto, Y. Ishii, I. Nishinaka, T. K. Sato, Y. Nagame, W. Sato, Y. Tani, H. Hasegawa, K. Matsuo, D. Saika, Y. Kitamoto, A. Shinohara, M. Ito, J. Saito, H. Kudo, A. Yokoyama, M. Sakama, K. Sueki, Y. Oura, H. Nakahara, M. Schädel, W. Brüchle, and J. V. Kratz, Radiochim. Acta 96, 125 (2008).

(13) Y. Ishii, A. Toyoshima, K. Tsukada, M. Asai, H. Toume, I. Nishinaka, Y. Nagame, S. Miyashita, T. Mori, H. Suganuma, H. Haba, M. Sakamaki, S. Goto, H. Kudo, K. Akiyama, Y. Oura, H. Nakahara, Y. Tashiro, A. Shinohara, M. Schädel, W. Brüchle, V. Pershina, and J. V. Kratz, Chem. Lett. 37, 288 (2008).

(14) H. Haba, D. Kaji, Y. Kudou, K. Morimoto, K. Morita, K. Ozeki, R. Sakai, T. Sumita, A. Yoneda, Y. Kasamatsu, Y. Komori, A. Shinohara, H. Kikunaga, H. Kudo, K. Nishio, K. Ooe, N. Sato, and K. Tsukada, Phys. Rev. C 85, 024611 (2012).

(15) M. Maly, T. Sikkeland, R. Silva, and A. Ghiorso, Science 160, 1114 (1968).

(16) S. Bouhlassa and F. Salhamen, Radiochim. Acta 101, 175 (2013).

(17) H. Kikunaga, Y. Kasamatsu, K. Takamiya, T. Ohtsuki, H. Yuki, A. Yokoyama, T. Nakanishi, and T. Mitsugashira, Appl. Radiat. Isot. 67, 539 (2009).

(18) H. Haba, K. Tsukada, M. Asai, I. Nishinaka, M. Sakama, S. Goto, M. Hirata, S. Ichikawa, Y. Nagame, T. Kaneko, H. Kudo, A. Toyoshima, Y. Shoji, A. Yokoyama, A. Shinohara, Y. Oura, K. Sueki, H. Nakahara, M. Schädel, J. V. Kratz, A. Türler, and H. W. Gäggeler, Radiochim. Acta 89, 733 (2001).

(19) CRC Handbook of Chemistry and Physics, $69^{\text {th }}$ ed., edited by R. C. Weast, M. J. Astle, and W. H. Beyer (CRC Press, Inc., Florida, 1988-1989) p.D-163.

(20) H. Bilinski, M. Branica, and L. G. Sillen, Acta Chem. Scand. 20, 853 (1966).

(21) J. Ueda and M. Takagi, Bull. Chem. Soc. Jpn. 63, 544 (1990).

(22) Stability Constants of Metal-Ion Complexes, Special Publication No.17, edited by L. G. Sillen and A. E. Martell (the Chemical Society, London, 1964).

(23) The Hydrolysis of Cations, C. F. Baes, Jr. and R. E. Mesmer (Wiley, New York, 1976). 